\title{
Has a new color area been discovered?
}

TO THE EDITOR - A recent paper in Nature Neuroscience 1 claims to show "a previously undifferentiated cortical area that we call V8" in the human fusiform gyrus. This claim has given hopes to some ${ }^{2}$ that the cortical area responsible for the conscious perception of colors in humans has at last been found. However, the Talairach coordinates for this 'new' area "V8" (ref. 1) are identical to those that we had published for V4 (ref. 3). The authors have therefore not found a new area; instead they have rediscovered and tried to rename area V4. Furthermore, their report ${ }^{1}$ states, in reference to our paper ${ }^{3}$, that "a prior study also concluded that this human color-selective region included a representation of upper and lower visual fields". How, then, can they state that colors activate "area V8 but not V4" (ref. 1)?
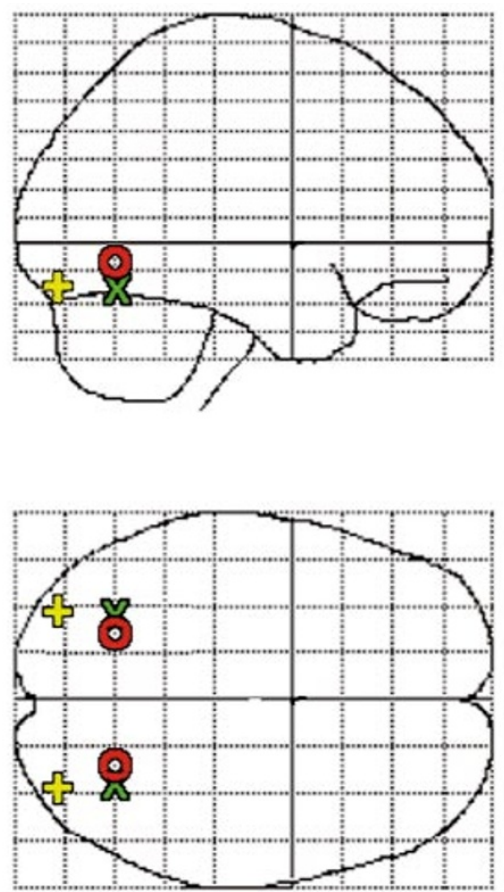

Fig. 1. The figure shows the locations of the three areas that are discussed in the text, in a glass brain projection. The areas were lo cated by using the Talairach coordinates of the three areas given in the paper by $H$ adjikhani et al. (1998): 0 corresponds to area V4 defined in Lueck et al. Nature $\mathbf{3 4 0}$, 386-389 (1989); Zeki et al. J. Neurosci. 11, 641-649 (1991); McKeefry and Zeki Brain 120, 2229-2242 (1997). X corresponds to the 'new' area ' $V 8$ ' of Hadjikhani et al. and the + to the area V4v defined by Sereno, M.I., et al. Science 268, 889-893 (1995).
The answer is simple: it hinges on the use of the letter $\mathrm{v}$, enabling one to write of $\mathrm{V} 4$ or $\mathrm{V} 4 \mathrm{v}$. To understand how a single letter can lead to such confusion, one has to retrace the history of the subject briefly. In 1995, Sereno and his colleagues, including Roger Tootell, co-author of ref. 1, reported the results of their mapping experiments in human visual cortex ${ }^{4}$. Many of the areas described had maps similar to ones found earlier in the macaque. Their map of what they supposed to be human V4 was not so straightforward. They distinguished a ventral V4v, located in the fusiform gyrus, from a dorsal V4d, located dorsolaterally, the two separated from each other by a relatively large expanse of cortex. V4v was clearly shown in the diagrams, but not V4d. This separation was unlike the V4 map in the monkey, where the two subdivisions, representing lower and upper visual fields respectively, are continuous with each other ${ }^{5}$.

This made us suspicious, because the clinical evidence shows that lesions in the fusiform gyrus, where we had located V4 (refs 3,6) can result in total hemi-achromatopsias ${ }^{7,8}$ that include both upper and lower quadrants of the visual hemifield. We therefore undertook a mapping experiment ${ }^{3}$ and found, unlike the Sereno report ${ }^{4}$, that both quadrants are mapped within the color center (area V4) in the fusiform gyrus of each hemisphere. Human V4, like monkey V4, therefore contains a complete map of the visual hemifield in each hemisphere. It is this crucial finding that Hadjikhani et al. ${ }^{1}$ have now confirmed. Not surprisingly, the Talairach coordinates of their 'new' area are identical to those of V4 $( \pm 26,-67,-9$ for our V4 and $\pm 33,-65,-14$ for the 'new' color area) but differ significantly from the coordinates of the more posterior $\mathrm{V} 4 \mathrm{v}$, at $\pm 32,-87,-16$ (Fig. 1).

Now we can see how one can write that color selectivity "is located in area V8, rather than in 'V4"' (ref. 1) or pretend that cerebral achromatopsia is produced by lesions of "area V8, not the favorite candidate V4" (ref. 2). One can do so by simply dropping the $\mathrm{v}$ from the $\mathrm{V} 4 \mathrm{v}$ and not stating explicitly that the human color center is distinct from $\mathrm{V} 4 \mathrm{v}$, though identical in position to V4 (refs $3,6)$. Both the above quotes would be correct, if the $\mathrm{v}$ were reinstated into the V4; both are wrong without it, as is the statement that "the human color center is distinct from area V4" (ref. 1). Whether this attempted renaming solves any of the mysteries of conscious color perception remains to be seen.

By stating that "color-selective activity is located in area V8 rather than in 'V4'" (without adding the v), they have misled readers into believing that they have identified a new color area ${ }^{2}$. That is the central issue that the authors ought now to address unambiguously, by acknowledging that they have not discovered a previously undescribed area, that color activates V4 selectively but not $\mathrm{V} 4 \mathrm{v}$, and that the coordinates of the human color center coincide with what we have given for V4 (refs 3, 6) but differs from that of their V4v. We leave it to them to describe in another context their area $\mathrm{V} 4 \mathrm{v}$, which is the area that seems to have no monkey equivalent and thus represents the real new discovery.

\section{S. Zeki, D. J. McKeefry, A. Bartels and R. S. J. Frackowiak} Wellcome Department of Cognitive N eurology, Institute of N eurology, University College London, London WC1E 6BT, UK

Tootell and Hadjikhani Reply Macaque V4 is a cortical visual area that is often subdivided into dorsal and ventral parts ('V4d' and 'V4v'), comprising the retinotopic representations of the lower and upper visual fields, respectively. This has been reported in numerous articles and is not in dispute. Nevertheless, Zeki and colleagues make the following claims: (1) human area $\mathrm{V} 4 \mathrm{v}$ "seems to have no monkey equivalent, and thus [it] represents the real new discovery," and (2) our human area $\mathrm{V} 8$ is actually equivalent to macaque V4. Their claim is based on three properties that they require of human V4: (3) human V4 should include a representation of both upper and lower visual fields, (4) human V4 should be colorselective and (5) human V4 coincides with the neural region affected in clinical achromatopsia.

Our response to each of their five points follows. (1) Human V4v is not a newly discovered area, as claimed by 